\title{
3D STRUCTURE ANALYSIS: ARCHITECTURE AS AN EXPRESSION OF THE TIES BETWEEN GEOMETRY AND PHILOSOPHY
}

\author{
Tamara Bellone $^{1}$, Luigi Mussio ${ }^{2}$, Chiara Porporato ${ }^{1}$ \\ ${ }^{1}$ DIATI, Politecnico di Torino, Torino, Italy , tamara.bellone@polito.it/chiara.porporato@gmail.com \\ ${ }^{2}$ DICA, Politecnico di Milano, Milano, Italy, luigi.mussio@polimi.it

\section{Commission II}

KEY WORDS: Euclidean Geometry, Projective Geometry, Non Euclidean Geometries, Mathematics and Philosophy, Mathematics and Architecture, Philosophy of Architecture.

\begin{abstract}
:
In recent decades many Geomatics-based methods have been created to reconstruct and visualize objects, and these include digital photogrammetry, Lidar, remote sensing and hybrid techniques. The methods used to process such data are the result of research straddling the fields of Geomatics and Computer Vision, and employ techniques arising from approaches of analytical, geometric and statistical nature. One of the most fascinating fields of application concerns Architecture, which, moreover, has always depended on Mathematics generally and, more specifically, on Geometry.

Throughout history the link between Geometry and Architecture has been strong and while architects have used mathematics to construct their buildings, geometry has always been the essential tool allowing them to choose spatial shapes which are aesthetically appropriate.

Historically, mathematics and philosophy have been interrelated; many philosophers of the past were also mathematicians.

The link between Philosophy and Architecture is twofold: on the one hand, philosophers have discussed what architecture is, on the other, philosophy has contributed to the development of architecture.

We will deal with the ties between Architecture, Geometry and Philosophy over the centuries. Although there are artistic suggestions that go beyond time and space, and there are genial precursors, we can identify, in principle, some epochs: the ancient era, the modern era, and finally the contemporary epoch, from the crisis of positivistic sciences to globalisation.
\end{abstract}

\section{INTRODUCTION}

Architecture, Geometry and Philosophy are connected by some interrelations. Indeed, throughout history the link between Geometry and Architecture has been strong and while architects have used mathematics to construct their buildings, geometry has always been the essential tool allowing them to choose spatial shapes which are aesthetically appropriate. The link between art and mathematics is recognizable in the works of painters and architects throughout history.

Historically, mathematics and philosophy have been interrelated; many philosophers of the past were also mathematicians. Plato went as far as saying that without mathematics there would be no philosophy as mathematics was a precondition for its birth. The truth of mathematics is not guaranteed by an external authority: mathematics introduces a universality free from mythological and religious assumptions, because it depends on rational and refutable demonstrations (Badiou, 2017).

Descartes introduced mathematical reasoning, but also the reductio ad absurdum into philosophy, while Spinoza's texts resemble mathematical proofs, and Leibniz invented infinitesimal calculus. Kant also believed that mathematics was indispensable to philosophy, and his conception of mathematics is of the a priori type.

The link between Philosophy and Architecture is twofold: on the one hand, philosophers have discussed what architecture is, on the other, philosophy has contributed to the development of architecture

An important stage in the relationship between philosophy and architecture opens with the publication of Kant's Critique of Judgment. Previously, the only architectural theorists in the West had been Vitruvius and Leon Battista Alberti.

Currently, the Philosophy of Architecture is a branch of the Philosophy of the arts dealing with aesthetics, semantics and the relationship between Architecture and culture.

Below, we will deal with the relationship between Architecture, Geometry and Philosophy over the centuries.
Although there are artistic suggestions that go beyond time and place, and there are genial precursors, we can identify, in principle, some specific epochs:

$>\quad$ The ancient era is characterized by Euclidean geometry, the birth of western philosophy, and proportion and harmony in Architecture, obtained through the golden ratio amongst other mathematical approaches. This period goes up to and includes the Romanesque.

$>$ The construction of perspective and projective geometry, rationalistic philosophy linked to the birth of modern science, characterize the second architectural period (modern era), whose styles range from Gothic to Baroque, through the Renaissance. During this period, the structure, often hidden, of the building takes on great importance (Odifreddi, 2017); indeed, many architects are also mathematicians.

$>\quad$ The departure from Euclidean geometry, initiated by the development of projective geometry, continued from the mid-eighteenth century, with a reflection on the logical foundations of geometry, which led to the analysis of Euclid's fifth postulate (Stewart, 2007). Non-Euclidean geometries, topology, the discovery of n-dimensional space, on the one hand, and the crisis of Positivism and the Sciences, along with the Theory of Relativity on the other, had an overwhelming impact on art and architecture in the third epoch.

$>$ Nowadays, Mathematics is everywhere, innovative media are based on binary language and on more and more innovative algorithms, but there is no link between mathematics and philosophy (Badiou, 2017). On the contrary, mathematics and computing are linked to contemporary art ( computer art), and are an important part of architectural projects. 


\section{FROM ANTIQUITY TO THE RENAISSANCE: THE GOLDEN RATIO}

The Pythagoreans believed that the universe was founded on numbers. The main empirical support to Pythagorean concepts came from music: there is a connection between harmonic sounds and numerical ratios. If a string produces a note of a certain tone, a mid-length string produces a very harmonious note, called an octave.

But one of Pythagoras' followers, Hippasus of Metapontum, proved that the diagonal of a unitary square cannot be expressed in an exact fraction: the discovery of irrational numbers was devastating for the Pythagoreans.

A solid is regular (platonic) if it has equal rectangular polygon faces, positioned in the same way in the vertices. The Pythagoreans associated solids (tetrahedron, cube, octahedron, dodecahedron and icosahedron) to the four primary elements (earth, air, water, fire) and quintessence.

Euclid proved that there are no other regular solids. The dodecahedron and icosahedron call the pentagonal; if we inscribe a five-pointed star in the pentagon, the relationship between the star's side and the pentagon side is an irrational number: 1,618 ...

Remember that we denote the golden number by $\varphi$ (in honour of Fidias). If a segment $\mathrm{AB}$ is divided into two parts, such as : $\mathrm{AB}: \mathrm{AC}=\mathrm{AC}: \mathrm{CB}$, point $\mathrm{C}$ divides the segment in the so called golden ratio. The number $\mathrm{AB} / \mathrm{AC}$ is the golden number. Where $\mathrm{AB}$ is equal to $1, \varphi$ is 1.618590347 . Indeed:

$$
\begin{aligned}
& \frac{x}{1}=\frac{1}{x-1} \\
& x=\frac{1+\sqrt{5}}{2} \cong 1.618
\end{aligned}
$$

Thus, Euclid studied regular solids in order to study irrational numbers.

Since antiquity this proportion has been considered a symbol of the harmony and beauty of the universe, and Kepler, indeed, came to believe that the order of the universe was based precisely on divine proportions: "I am convinced that this geometric proportion served from the idea to the Creator, when $\mathrm{He}$ introduced the continuous generation of shapes similar to each other." Johannes Kepler (1571-1630) was very interested in the golden ratio. He wrote, "Geometry has two great treasures: one is the theorem of Pythagoras, the other the division of a line into mean and extreme ratios, that is $\Phi$, the Golden Mean. The first way may be compared to a measure of gold, the second to a precious jewel."

Indeed, Kepler's triangle (Figure 1) combines these two mathematical concepts - the Pythagorean theorem and the golden ratio. Kepler first demonstrated that this triangle is characterised by a ratio between short side and hypotenuse equal to the golden ratio.

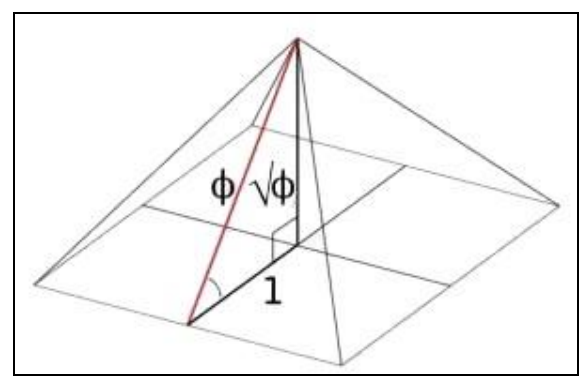

Fig.1 Kepler's triangle

A golden ratio pyramid is based on a triangle whose three sides represent the mathematical relationship that defines the golden ratio. The golden section and architecture have a long historical relationship: the ancient Egyptians and ancient Greeks incorporated this and other mathematical relationships, such as the 3:4:5 triangle, into the design of monuments including the Great Pyramid and the Parthenon. The Cheops pyramid has the following dimensions: height of about $146.5 \mathrm{~m}$, the other cathetus of $115.2 \mathrm{~m}$ (the side of the square is $186.4 \mathrm{~m}$ ): the hypotenuse is therefore $1,864 \mathrm{~m}$ If we give the minor cathetus a value of 1 , we obtain the following ratio: 1.27 : 1 : 1.61 ; since the square root of 1.61 is 1.27 , we can deduce that in the construction of the Cheops pyramid the golden section was applied (Figure 2).

In fact, the angle between the base and the cathetus of a 3:4:5 rectangular triangle is $3 / 4$, that means a $75 \%$ slope $\left(53^{\circ}\right)$ : actually Cheops pyramid has a $51^{\circ}$ slope.

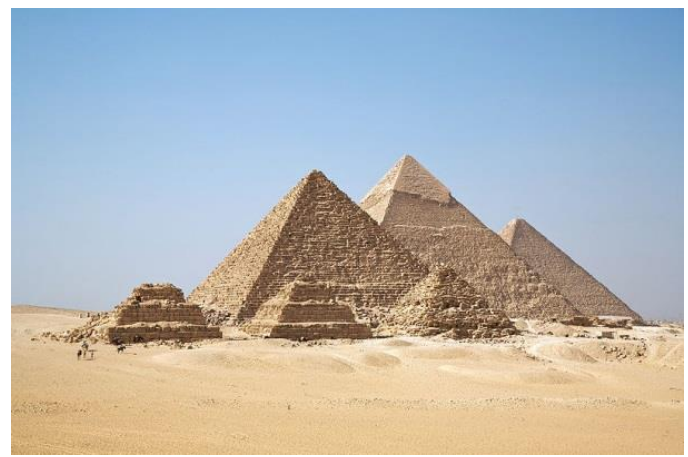

Fig. 2 Cheops pyramid

Pre-Columbian stepped pyramids (Figure 3) also have square base, while Guimar (in Tenerife) pyramids have a rectangular base, as do ziggurats (Figure 4).

The golden ratio, for the very reason that it is seen as an ideal of beauty and harmony, is also found in many classical Greek sculptures, such as the Doryphoros by Polykleitos.

Homer knows hybris (v́ßpı): at the beginning of the Iliad, Achilles shows a deathly anger, as Agamemnon has taken for himself, with arrogance and violence, his prey, Briseid.

Nemesis is the punishment for hybris; the opposite of hybris is the sense of limits (the sense of limits is well known also in politics, as in the case of Solon).

The sense of limits is clearly linked to harmony, symmetry and proportion.

Ancient Egyptians and Greeks had a long-lasting intercourse: Pythagoras discovered in Egypt his measuring techniques: thus, geometry and numbers were associated from the beginnings of history.

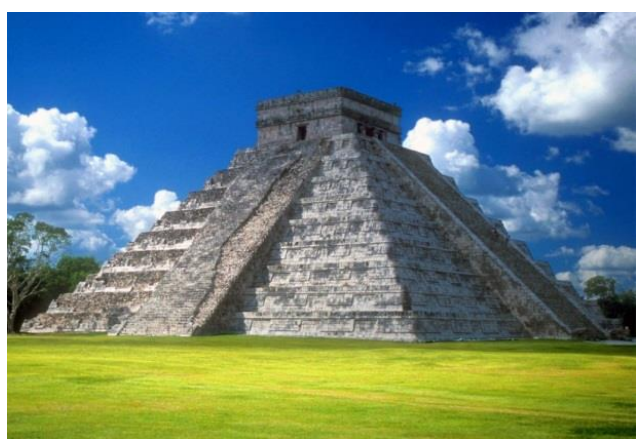

Fig. 3 El Castillo pyramid, Chichen Itza, Mexico

Numbers were matched to divinities (the Egyptian god Thot, or Prometheus, Uranus,...), so that geometry had, at certain times, a sacred value (Zellini, 2016). The Greeks thought of celestial movements as precise and perfect, but also said that perfection is not of our world, which is inexact and ruled by "more-or-less" situations (Koyré,1967). Similarly, in 
morality: mathematical approximations to an irrational number resemble excess and deficiency in our moral life (Zellini, 2016).

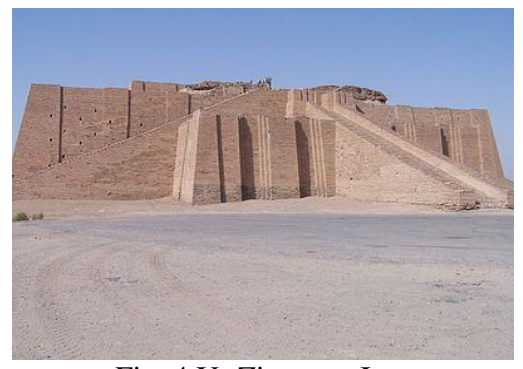

Fig. 4 Ur Ziggurat, Iraq

In contrast, the association of the material world of forms and the abstract world of numbers may be the origin of the link between natural sciences and mathematics, which in turn is the basis of Newton's physics in the $17^{\text {th }}$ century (Capra, Mattei, 2017). However, art is an access to the world of exactness and perfection, since art evokes cosmic order.

Golden rectangles are observable on the façade of the Parthenon (Figure 5). In the Parthenon, the overall height is the golden section of the width of the front part; thus, the facade has the size of a golden rectangle. This golden ratio is repeated several times between different elements of the front, for example, between the overall height and the height of the entablature.

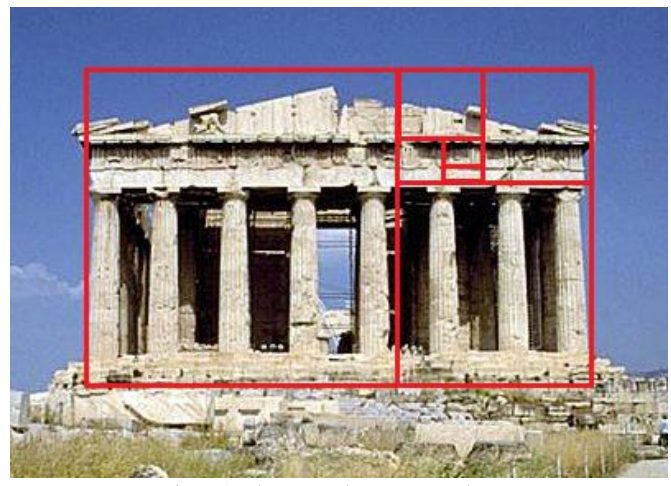

Fig. 5 The Parthenon, Athens

It might indeed appear that constructions designed using the golden ratio are based on an aesthetic archetype: Puerta del Sol near La Paz, for example, is based on golden rectangles (Figure 6). In 1876, Gustav Fechner (1801-1887), the inventor of Psychometry, performed a statistical test on a number of people without any artistic background, asking them to choose one of a number of rectangles: the most frequent choice was a golden rectangle.

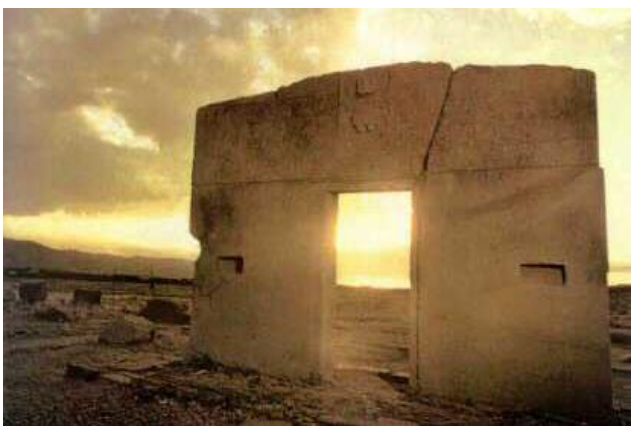

Fig. 6 Puerta del Sol, Teotihuacan, Bolivia
The "most beautiful relation" (according to Plato's Timaeus) is also present in the theatre at Epidaurus (Figure 7), built by Polykleitos the Younger. The stage is surrounded by a first group of 34 steps; thereafter a double-step space, a second group of 21 steps is present; the ratio $34 / 21$, which is to say 1.619 , as $55 / 34$ is to say 1.617 .

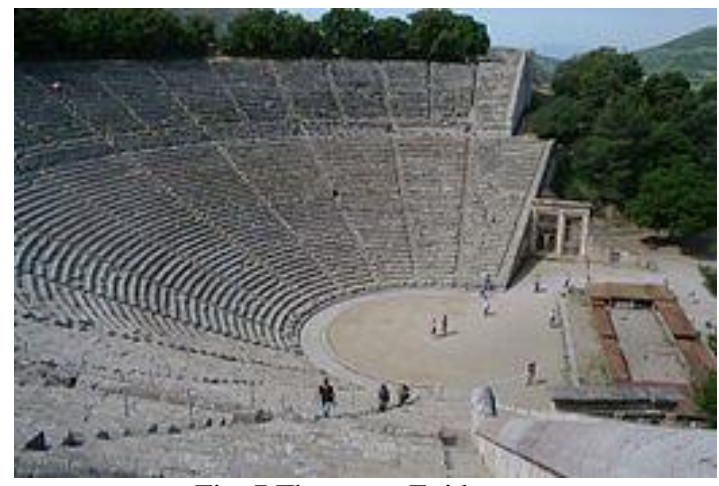

Fig. 7 Theatre at Epidaurus

Also, in a regular pentagon, some $\varphi$ ratios are present: two diagonals without a common vertex are mutually bisected into golden proportions. In a regular pentagon, too, the diagonal and one side have a golden ratio.

Diagonals of a regular pentagon, cross in the shape of a fivevertex star, plus a smaller pentagon. The procedure can be iterated indefinitely, as proved by Hippasus of Metapontum. Military engineers have generally used a pentagon (Figure 8) in the design of fortresses (see also the Pentagon at Washington, DC). Even in several, important paintings, an underlying pentagonal and five-pointed star structure may be discerned.

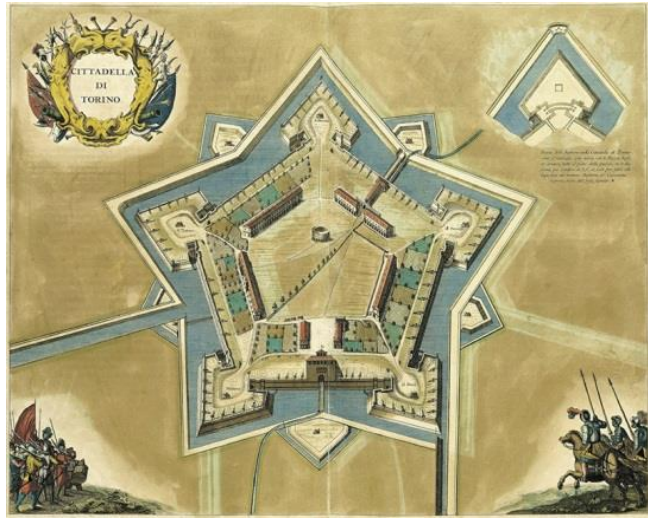

Fig. 8 The design of the citadel of Turin

Indeed, Euclidean geometry informed architectural styles right up to the Romanesque period. The Sectio aurea was of great interest during the Renaissance, from Leon Battista Alberti to Leonardo da Vinci to: one charming example (Figure 9) of such is Sant' Andrea Church in Mantua (Leon Battista Alberti,1460).

The most important architectural theory treatise is "De Architectura" by Vitruvius, according to whom the architect must know philosophy in order to be motivated not only for practical purposes but also for aesthetics.

The architect must build taking into account stability (firmitas), utility (utilitas) and beauty (venustas).

The idea of Vitruvius, and later of Leon Battista Alberti and others, is that beauty, born of proportion and symmetry, is the result of an underlying ideal reality in which there is regularity and order. 


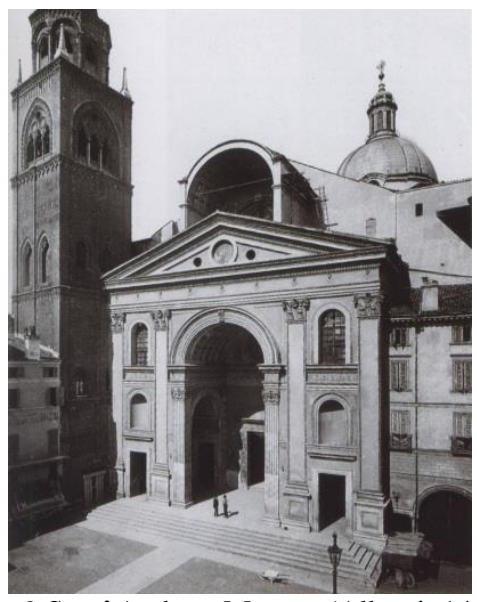

Fig. 9 Sant' Andrea, Mantua (Alberti, 1460)

\section{THE MODERN ERA: THE BIRTH OF THE INFINITE}

Thomas Aquinas says that a thing is beautiful not because it is pleasing but because it is beautiful in itself. Each thing is beautiful in so far as it manifests: wholeness (integritas), harmony (consonantia) and radiance (claritas) (Haldane, 1999).

Panofsky proposes a parallel between the Gothic cathedral and scholastic philosophy: both aspire to totality, articulation and coherence. The expressive architectural form, typical of the gothic style, reflects and is built thanks to a precise mental habit, articulated according to the principles of scholastic philosophy (Panofsky, 2016).

Renaissance artists invented perspective, in order to more realistically represent the three dimensions on a flat surface.

The construction of perspective remains within the domain of Euclidean geometry. Brunelleschi and Leon Battista Alberti (De pictura, 1435) simplified human vision by imagining that the artist saw the scene from a single eye. The relation of parallelism is not maintained, in fact if we are the center of a road we see that the edges, in parallel relatives, meet in a single point, on the horizon: the core of the technique lies in the vanishing point placed at infinity.

Central perspective is often considered natural and immediate. According to Erwin Panofsky (Panofsky, 1961), however, space as conceived by Brunelleschi and Alberti is mathematical and rational, preceding the analytic geometry of Descartes and the Copernican revolution: perspective is a symbolic construction (this concept was introduced by Cassirer).

The artists who painted the orthodox icons could never have invented perspective, as they had to place Christ in a spiritual and unrealistic space. In this regard, we compare three paintings on the same theme, the crucifixion of Christ: an orthodox icon, a Renaissance painting and a painting by Chagall, which deliberately returns to anti-perspective (Figures 10, 11 and 12).

In ancient times the purpose of natural philosophy was to understand nature in order to live in harmony, while from the seventeenth century onwards the philosophy of nature turned into the science of nature and its purpose became to use science to use nature, not to understand it: the world was res extensa, while man was outside nature, res cogitans.

Copernicus, Tiho Brahe, Cardano are referable to the artistic Renaissance, but the new science (Galileo, Kepler, Descartes, Harvey, Huygens, ...) does not have the same vision of the Baroque. Indeed the ambiguity, illusion, emotionality and sensoriality inherent in Baroque, do not coincide with the values of the new science: clarity, truth, rationality
Some artists began to introduce ambiguity between constructed space and perceived space, starting with anamorphosis.

Generally, architects and painters of the $16^{\text {th }}$ century used, particularly for ceiling frescos, a series of vanishing points in order to limit the paradoxical effects of perspective. Some innovators, such as Niceron, Maignan and Andrea del Pozzo, used a unique, central viewpoint which resulted in significant distortion at the edges.

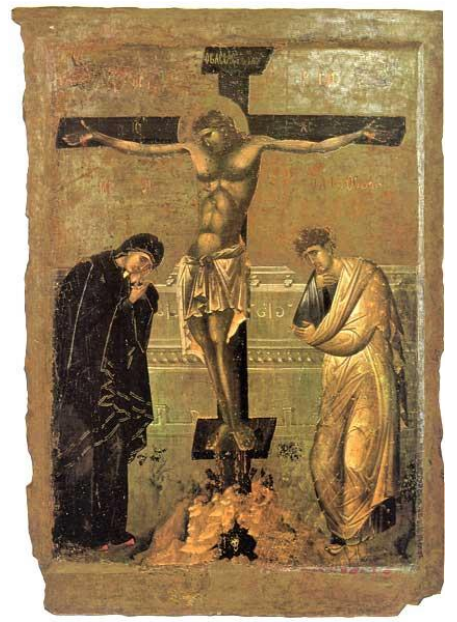

Fig. 10 The crucifixion, $\left(14^{\text {th }}\right.$ century $)$, at Ohrid

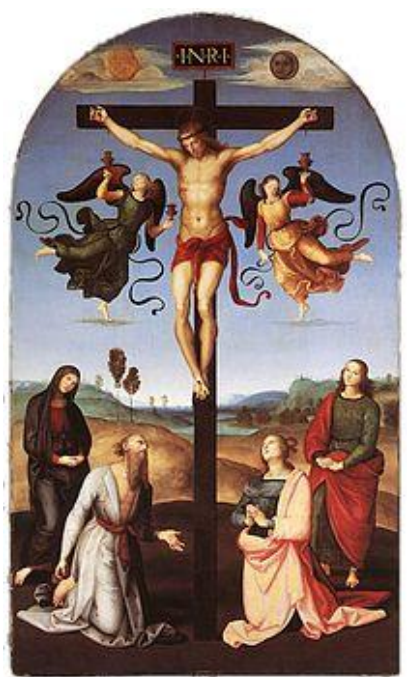

Fig. 11 The crucifixion, Raphael (1520)

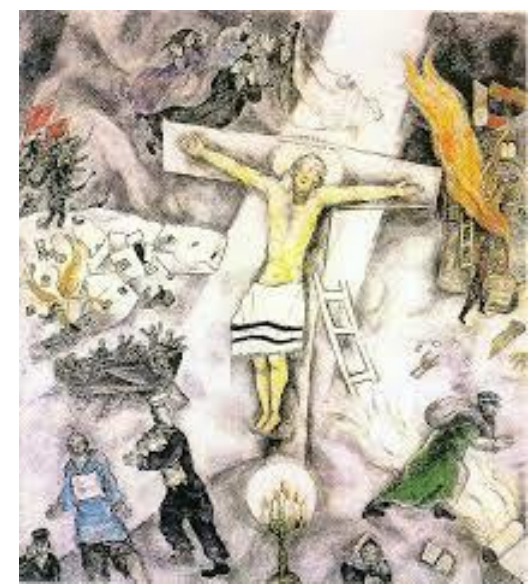

Fig. 12 The white crucifixion, Chagall (1938) 
Moreover, the quadrature, which developed from the sixteenth century onwards, together with the studies on perspective, created the illusion of spaces different and broader than the real: there are numerous 'fake' vaults in baroque churches (Figure 13).

Baroque architecture is linked to the projective geometry and mathematics of the time. In the meantime, in fact, a new type of geometry, later called projective, had developed from the studies of the architect Desargues. This new geometry does not negate the presuppositions of Euclidean geometry, but it extends its boundaries: in fact it allows us to represent the infinite through the improper points.

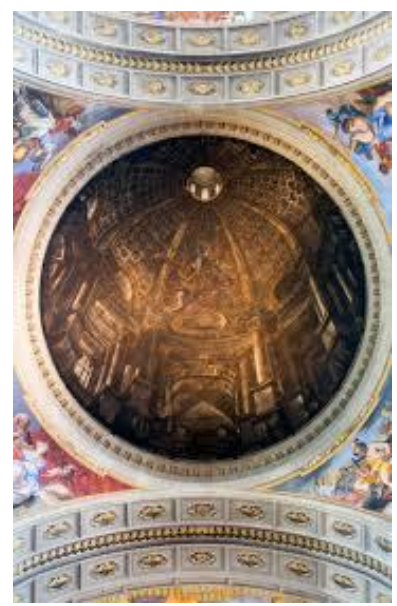

Fig. 13 False dome, Sant'Ignazio di Loyola, Rome (Andrea Pozzo,1685)

Projective geometry rediscovered conics, Baroque architects used them in an innovative way compared to the ancient Greeks. Moreover, the design of these structures was made easier by the introduction of descriptive geometry, which permitted the design of the volumes.

Desargues was an architect and a mathematician, he wrote an essay on the results of taking plane sections of a cone" (Brouillon proiect d'une atteinte aux evenemens des rencontres du cone avec un plan).

Shapes and sizes change according to the plane of incidence that cuts the cone of the projection, but certain properties remain the same throughout such changes, or remain invariant under the transformation, and it is these properties that Desargues studied. Conic sections, including parabolas, ellipses, hyperbolas, and circles, can be obtained by continuously varying the inclination of the plane that makes the section, which means that they may be transformed into one another by suitable projections and are therefore continuously derivable from each other (Figure 14).

Space is considered to be that which enables such transformations. In other terms, perspective produces a series of corresponding shapes which are very different from the originals. Nonetheless, they share some proportional properties based on the involution relation. The dissimilitude between the projected objects and their originals appertains to the relative positions of the eye (or the vertex of a cone) and the projection plane (Debuiche, 2012).

According to Leibniz, each monad expresses the world from its own particular point of view. He often refers to the transformations of projective geometry, which he had learned directly from Desargues.

Even Leibniz, like many other thinkers of the seventeenth century, tries to find a solution to the problem inherited by Descartes on the relationship between res cogitans (spirituality) and res extensa (materiality): Leibniz will find a solution to the question diametrically opposed to that of
Hobbes, arguing that there is only res cogitans: the only existing reality, for the German thinker, ends up being the spiritual reality and what we commonly call "matter" is nothing but a secondary manifestation of res cogitans.

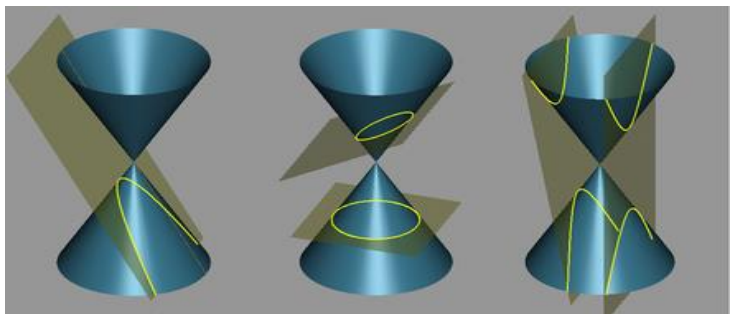

Fig. 14 Conics

If the monads are representations of reality, they must necessarily also be perceptions of reality, that is, ways of grasping what in reality takes place. Each monad, as we said, has perception (because it represents) of the universe from a single point of view, but the monad that counts most of all (God) collects in itself all the infinite points of view on the universe. Appearances, on the other hand, are perceptions endowed with self-awareness. Man is endowed with it, but also many other animals: for Leibniz there is not that clear distinction between man and animal asserted by Descartes. In other words, those who have apperceptions not only represent the world, but also have the awareness of perceiving it.

For Newton, in contrast, first there is space and time, and then, later, other things: and moreover it is impossible to imagine bodies without resorting to space. Space for the English thinker ends up being a sort of container in which all things and subjects are perceived, a container that was born before and that would continue to exist even if there were no longer things and subjects that occupy it. For Leibniz, however, before space and time there are things, which he calls monads, and space and time are simply relations that are established between the same: they are the material monads that establish reciprocal relationships giving life to space; in the same way, time is the succession of states of the monads. Caramuel and Guarini were mathematicians as well as architects (and ecclesiastics).

Juan Caramuel y Lobkowitz (1606-1682) traveled across Europe ending up, as a punishment, in Vigevano. He is considered one of the forerunners of binary calculation and the inventor of oblique architecture, he adhered to the philosophical probabilism, according to which conscience could prevail, in certain cases, over religious doctrine.

Caramuel's concept is distant from that of Bacon and Descartes: for him the world is not mechanistic, but complex and full of interactions and connections.

The façade of the Cathedral of Vigevano (Figure 15 )is oblique and strongly offset from the axis of the cathedral and also from the axis of the square, and it opens onto the square like a stage.

Gradually, we have passed from circular designs to the oval and then to elliptical ones: how then could we not think of Kepler's celestial geometries?

In contrast, Guarini supports the thesis that all the arts depend on mathematics and philosophy: these sciences deal with relationships between objects and with their proportions; his mathematical-philosophical treatise Placita philosophica reveals the influence of Descartes' thought.

The works of Guarini are completely imbued with the aspirations of the Baroque style, for example, the structure of Guarini's Chapel of the Holy Shroud Dome (Figure 16) consists of 5 parts, the main cone in turn consists of six levels 
of triangles, tapering upwards, so as to obtain an illusion of greater height.

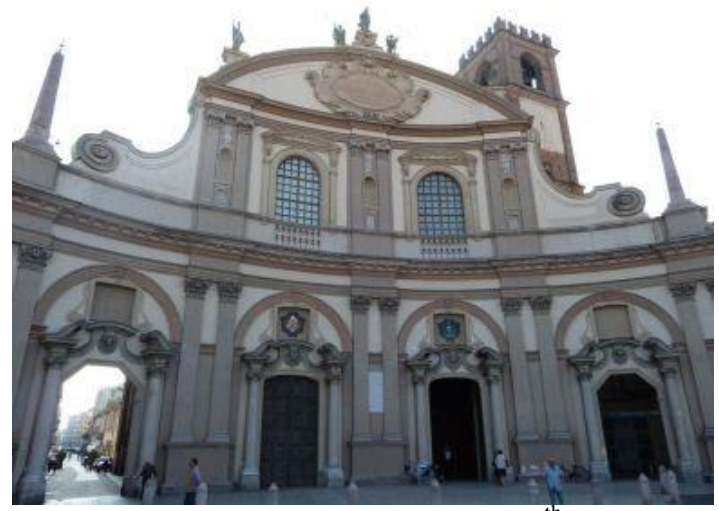

Fig. 15 The Cathedral of Vigevano, $17^{\text {th }}$ century

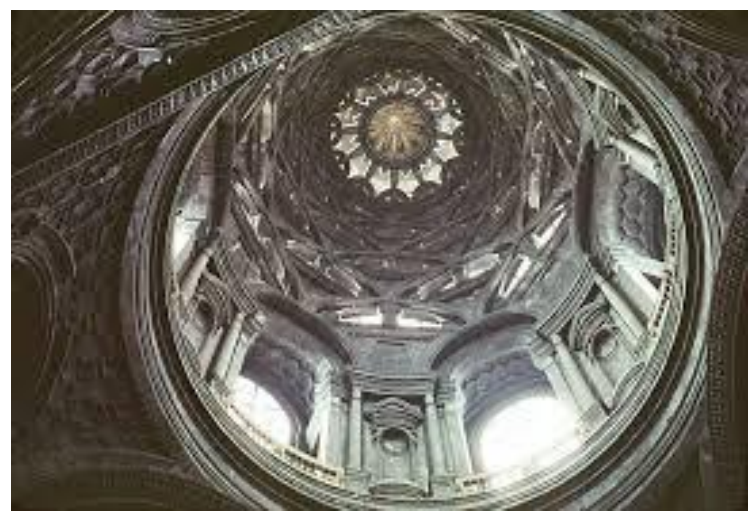

Fig. 16 The Chapel of the Holy Shroud, Turin (Guarini, 1668)

Moreover, the famous colonnade of St. Peter's Square by Bernini (Figure 17) reflects, in a certain sense, the principles of Caramuel's oblique architecture.

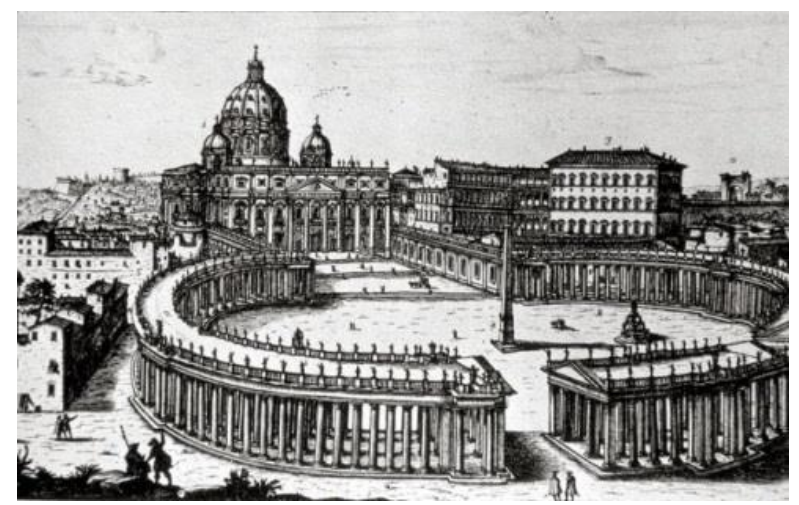

Fig. 17 colonnade of St. Peter's Square

What appears to be seen from one particular viewpoint, at the center of the hemicycle, gradually transforms as the observer proceeds towards the large portico: the columns swell, the bases and capitols are deformed, the pillars become oblique, above all in the areas on the margins, just like in the horrors of the artists who apply anamorphosis. (Figures 18a and 18b). The first anamorphosis depicts Saint Francis of Paula in prayer, under a tree: this image can be seen from the end of the corridor. If you stand directly in front of the fresco, the figure of the saint is deformed, while a marine landscape appears beneath him.

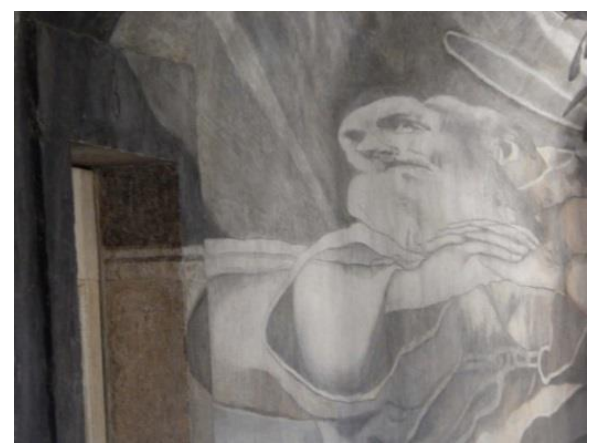

Fig. 18a fresco at Trinità dei Monti convent, Rome

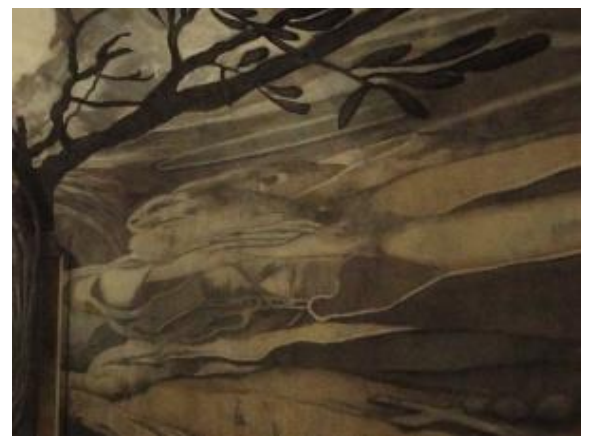

Fig. 18b fresco at Trinità dei Monti convent, Rome

Thanks to the rhythmic succession of concave and convex wavy parts and to the adoption of innovative and unusual designs, the Baroque buildings give the illusion that a fantasy world is opening up before the onlooker. (Figures19 and 20 ).

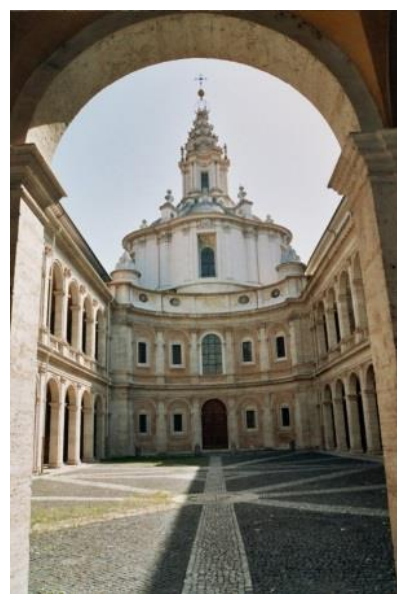

Fig. 19 Sant'Ivo alla Sapienza, Rome (Borromini, 1662)

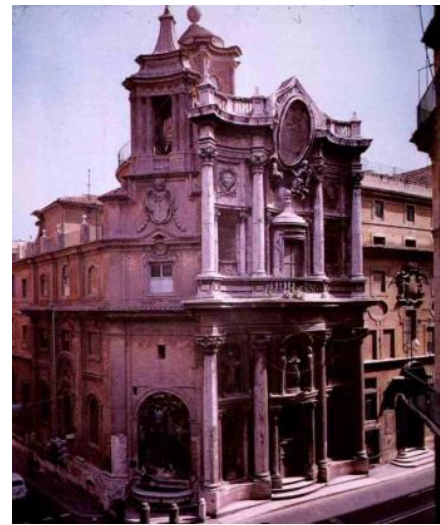

Fig. 20 San Carlo alle Quattro fontane, Rome (Borromini, 1667) 


\section{FROM CRISIS OF KNOWLEDGE, TO GLOBALIZATION}

Euler, had noticed that a surface in a three-dimensional space, described by an equation, could also be studied if a pair of coordinates, $\mathrm{u}(\mathrm{x}, \mathrm{y}, \mathrm{z})$ and $\mathrm{v}(\mathrm{x}, \mathrm{y}, \mathrm{z})$ upon the surface were to be associated: for instance, latitude and longitude upon a spherical surface.

Gauss, for his part, initiated "intrinsic" investigation of surfaces. Thus, in Euclidean space, a surface may be studied both "from outside", by its equation, and "intrinsically", having its local metrics described as intrinsic coordinates by a differential (Borzacchini, 2015)

In this respect, Gauss anticipated hyperbolic and elliptical geometries (Lobačevsky and Riemann).

It is easy to see that all triangles upon a sphere (i.e. the Earth, more or less...) have angles whose global amplitude is over $180^{\circ}$. Also, all geodesics meet at two points only. In this case, we can say that curvature is positive. Moreover, in the said geometry, parallels are quite absent. In contrast, for hyperbolic geometry, total amplitude of inner angles for triangles is less than $180^{\circ}$ and the curvature is described as negative: in this case, the number of parallels at a single point to a given geodesic is unlimited (Figure 21).

In an approach to the mathematical problem seeking a unitary understanding of different geometrical theories, as suggested by Klein, new developments in geometry also raise a philosophical question: what is the connection between geometry and the real world?

Cayley shows reciprocal connections between Euclidean and projective geometry, and it is due to him that, to this day, that the distinction is made between "elliptic" and "hyperbolic" non-Euclidean geometries, with normal Euclidean geometry defined as parabolic, seen as a transitional case.

All the aforementioned geometries may be taken to be special cases (or sub-geometries) of projective geometry, which proves independent from the Euclidean postulate of parallel lines (Stewart, 2016).

The curve perception of straight lines depends on the physiology of the eye: the retina is curved. Our eye experiences all three geometries Euclidean, spherical and hyperbolic (Odifreddi, 2011).

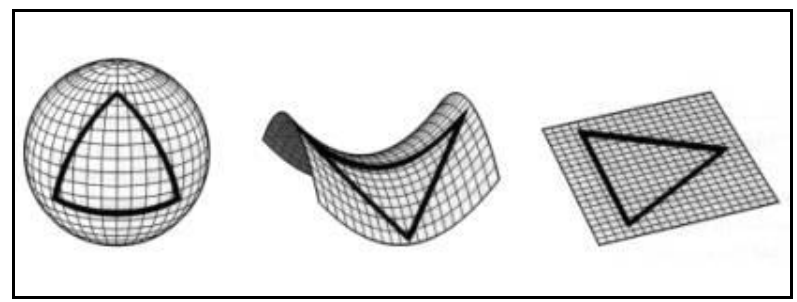

Fig. 21 Geodesics on different surfaces

For Poincaré too, mathematics has a dual nature, on one side it shares a border with philosophy, and on the other with physics.

Newton returns to Democritus' idea that space is an empty container, in which bodies move freely, until they encounter forces acting on them. Before Newton, the idea of a space as an entity separate from objects was not accepted, it was about extensive objects, that being was not separate from space, space was the extension, a prerogative of being (the res extensa of Descartes). In fact, Aristotle, himself, was already asking how, if there is nothing between one substance and another, space exists, if indeed it does. The philosophical issue was overshadowed by the success of Newtonian physics, which allows great practical results, but nonetheless remained in the background. And Einstein, initially influenced by Mach, reflected on the problem of being and non-being.

Furthermore, it was said that Newton's absolute space could be the gravitational field. The world no longer consists of particles in space, gravitational fields and magnetic fields, but only with particles and fields, it is not necessary to hypothesize absolute space. The gravitational field is therefore not within space, but it is space itself. Space is not a rigid container, but it bends, twists, is flexible. The Sun folds space around: the Earth then moves straight into a tilting space (Rovelli, 2014). Not only does space spin, but time too: Einstein feels that time runs faster higher up and slower, nearer the Earth. The story of the two twins is well-known: the one who lived in the mountains is a little older than the other one who lived at sea level.

For Kant, mathematics is universal not because it relates to reality, but because it relates to the universal cognitive structure, Kant's thesis is not similar to that of Wittgenstein, for whom mathematics is only one of the possible languages (he was interested in many different topics, e.g. Architecture, indeed he built a house for his sister as shown in Figure 22): for Kant mathematics is a transcendental type of formalism.

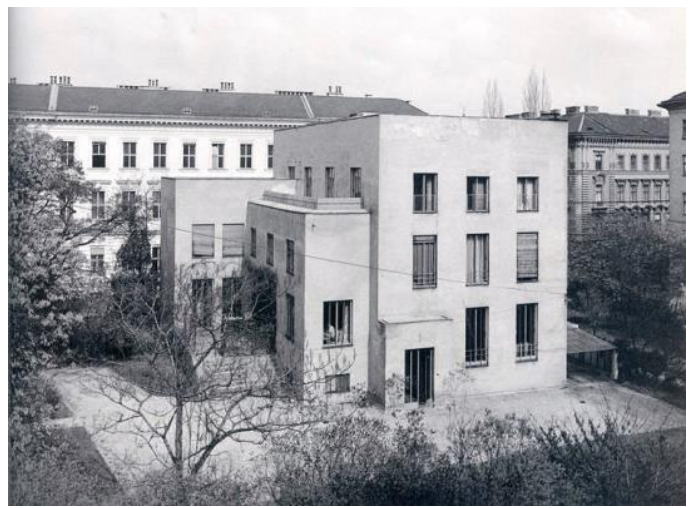

Fig. 22 Wittgenstein's house, (Engelmann and Wittgenstein, 1928)

The Kantian doctrine of space and time did not arise from psychological considerations, but mathematical and physical ones. Space and time are forms, that is, their reality is not that of things, but of conditions, of a priori presuppositions for all our empirical knowledge: space and time are objective without being absolute.

There is a great difference in the Philosophy of Architecture before and after Kant. According to Kant's Critique of Judgment (1790), Architecture like any other form of art, is considered an expression of abstract aesthetic ideas, and not merely a result of the pursuit of utility and beauty as it had been for Vitruvius. Indeed, for Kant the aesthetic judgment differs little from theoretical and moral judgments: all are "a priori”.

The concept of space and time, as forms of intuition- said Cassirer- does not require any change, in the perspective of the theory of relativity, provided that we continue to interpret them as conditions of every possible intuition.

Heisenberg says: "... At the heart of the question, there is always Kant's antinomy, as I have already said, so, on the one hand, it is very difficult to imagine that matter can be subdivided indefinitely, and on the other. it is equally difficult to think that this subdivision finds at a certain point, abruptly, an end. Ultimately, antinomy was born, as we now know, from the erroneous application of our intuition to the situations of the microscopic world. The greatest influence on the physics and chemistry in the last century has undoubtedly been exerted by Democritus' atomic theory; it allows for an intuitive description of chemical processes at the microscopic 
level. Atoms can be compared to the point masses of Newtonian mechanics and such a comparison leads to a satisfactory statistical heat theory. In fact, the atoms of the chemists were, at the time, not considered pointy masses, but small planetary systems; the atomic nucleus was composed of protons and neutrons, but it was thought that electrons, protons and possibly even neutrons could be considered as real atoms in the sense of the last indivisible bricks of matter. The atomic representation of Democritus thus became, for the last hundred years, an integral part of a physicist's general materialistic framework; it was easily understandable and rather intuitive, and also determined the scientific thinking of those physicists who did not want to have anything to do with philosophy. At this point, I would like to justify my hypothesis that in today's elementary physics, good physics is unknowingly ruined by bad philosophy. Use of a language that derives from traditional philosophy is inevitable. One wonders: 'What is the proton? Can the electron be subdivided or not? How light is simple or composed?' And so on. But all these questions are wrong, because the "split" or "consist of" expressions have largely lost their meaning. We should therefore adapt our problems, our language and our thoughts, that is our natural philosophy, to this new situation created by the experiments. But this is unfortunately very difficult. Thus, in the physics of particles, there are constantly misunderstood false questions and incorrect representations. [...]

If we want to compare the knowledge of current particle physics to some previous philosophy, this could only be Platonic philosophy; in fact, the particles of today's physics, as the theory teaches, are representations of symmetry groups, and are thus comparable to the symmetrical bodies of Platonic doctrine."

Note that the first signs of the twentieth-century revolution in art and architecture were, on the one hand, the Impressionists and their precursors (divisionists, macchaioli, symbolists, ...), on the other hand the Art Nouveau (Modernism, Jugendstil) and the Catalan school of Gaudì (Figure 23). Quadratic surfaces and catenary curves have been present in modern architecture only since Gaudì.

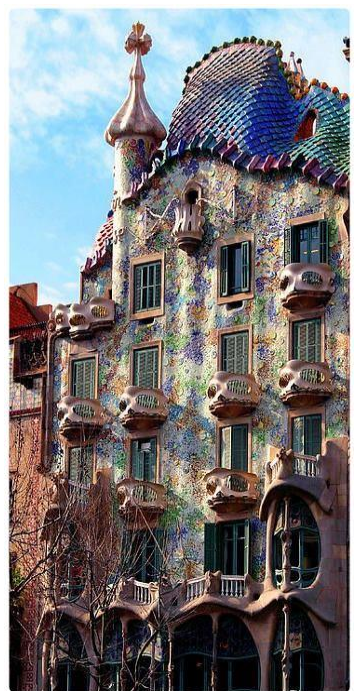

Fig. 23 Casa Batllò, Barcelona (1906)

The most famous exponent of the modern movement was Le Corbusier, according to whom architecture had to be humanfriendly. Indeed, Le Corbusier reawakened the interest in human proportions and Vitruvian rules, he created the Modulor (from module, unit of measure and or, golden section). The The High Court of Justice (Figure 24) is a mix of traditions and modernism.

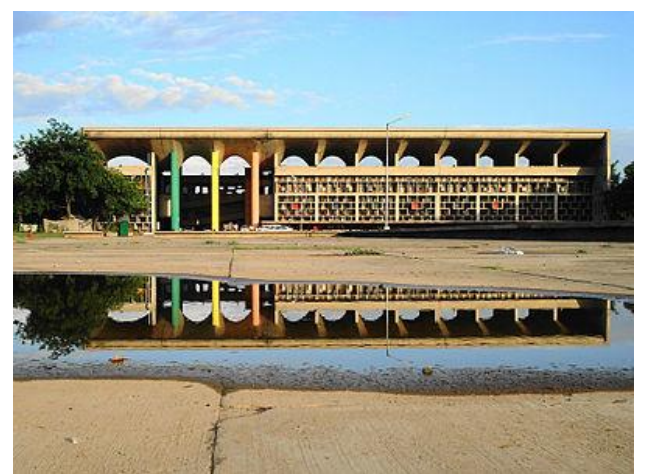

Fig. 24 The High Court of Justice, Chandigarh (1951-56)

The exploration undertaken by the so-called avant-gardes (Expressionism, Abstractionism, Cubism, etc.) began in 1907 with Les demoiselles d'Avignon by Pablo Picasso (Figure 25), and influenced all the arts, architecture above all.

Both non-Euclidean Geometry and the Theory of Relativity are at the root of Cubism. Cubist painting introduces a fourth dimension: time; in point of fact, one picture can have simultaneously different viewpoints, permitting analysis at different times (Figure 26).

The Einstein astronomic Observatory at Potsdam (1917-21) is convex and concave in its surfaces, with many openings. (Figure 27).

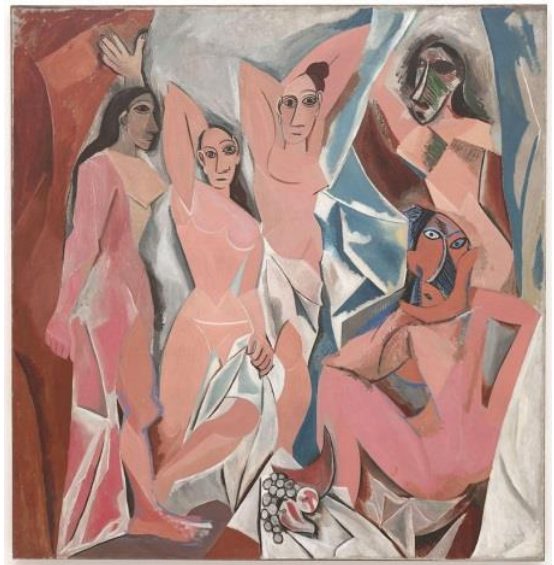

Fig. 25 Les demoiselles d'Avignon, (Picasso, 1907)

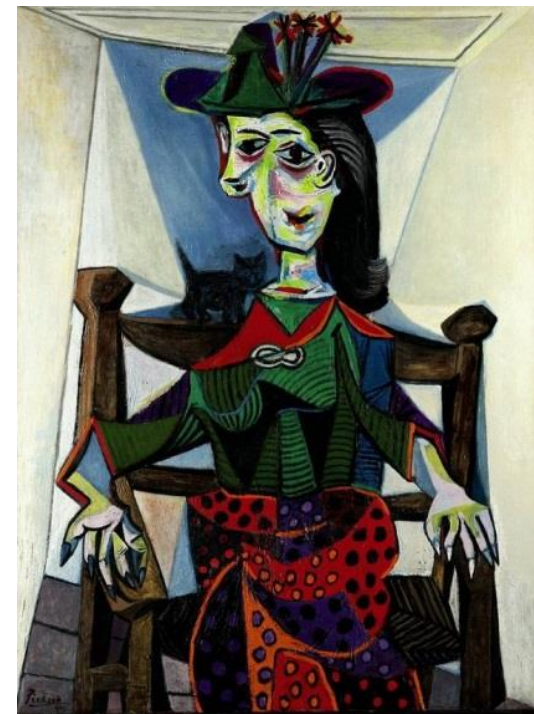

Fig. 26 Dora Mar au chat (Picasso, 1941) 


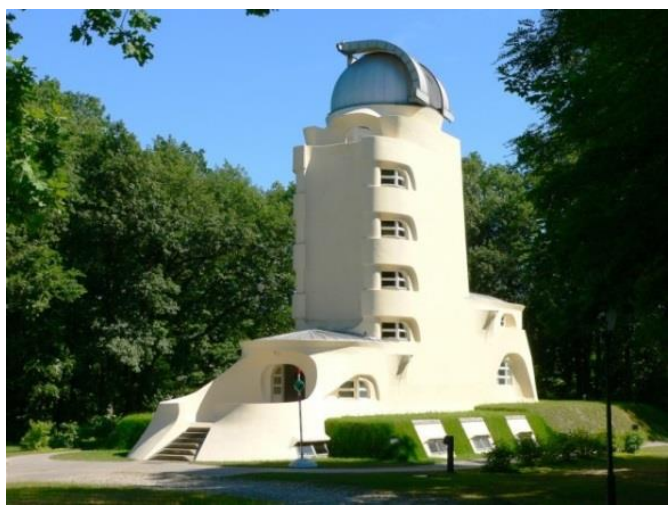

Fig. 27 Einstein astronomic observatory, Potsdam

After October 1917, despite its highly critical situation, with civil war and famine all around, the Soviet Union displayed a sudden flowering of artistic trends: actually, Anatolij Lunačarskij as the minister of Culture, granted great freedom to artists. Consequently, two trends emerged: pure, abstract art represented by Kandinsky and Malevich. (Figure 28), and a more realistic art, linked to social needs (Tatlin, Rodchenko), known as Constructivism.

The most representative result is the design for a monument to the $3^{\text {rd }}$ International $(1919-20)$ by Tatlin, which was however cancelled for economic reasons: it should have been a steel skew structure, able to support a mobile glass cover (Figure 29).

Lissitzky conceived the idea of building a cluster of horizontal skyscrapers in Moscow (Figure 30), but this idea was never realized.

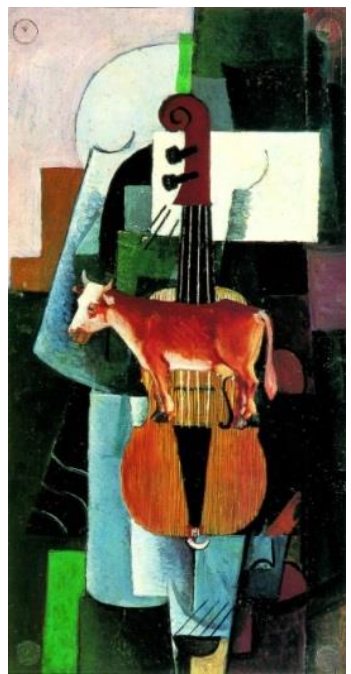

Fig. 28 Cow and violin (Malevich, 1913)

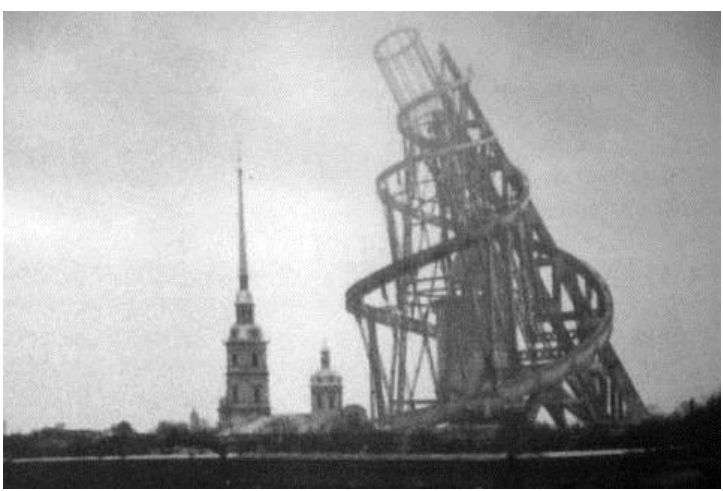

Fig. 29 The monument to the third International (Tatlin)

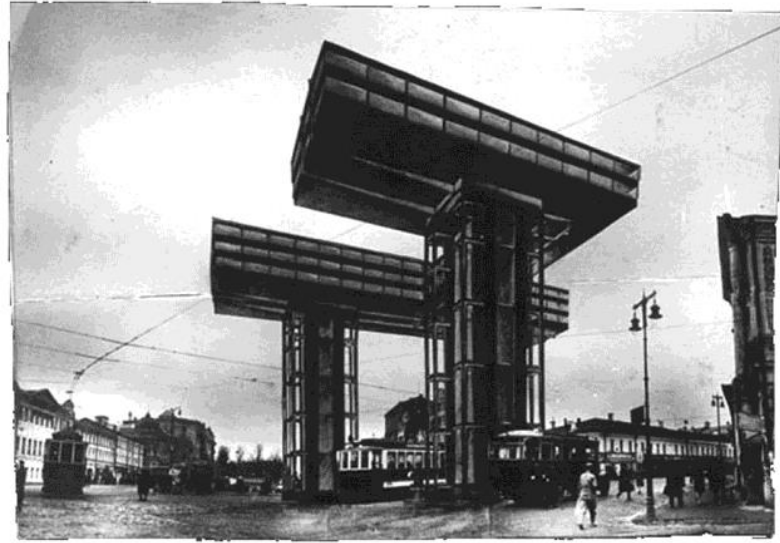

Fig. 30 Horizontal skyscraper (1925)

After 1924, an authoritarian political trend suffocated Constructivism, and in 1932, Soviet architecture returned to Classicism, a typical effect of reactionary involution (Montanari, 2017).

Globalized society has lost touch with the strong views of the previous two centuries.

In the nineteen-eighties, Derrida thought that the time had come, to take care of hidden aspects, even in architecture (Deconstructivism). So, new buildings began to appear, far removed from old rules, as a superimposition of diverse volumes, which appear as interpretable in different ways.

The Deconstructivistic Architecture Exhibition at New York (1988) shows plans by Frank O. Gehry (Figure 31), Peter Eisenman. Danile Libeskind, Zaha Hadid (Figure 32), Bernard Tschumi, Rem Koolhas and the Coop Himmelbau from Wien. They all pay due attention to the Russian experiences of the twenties.

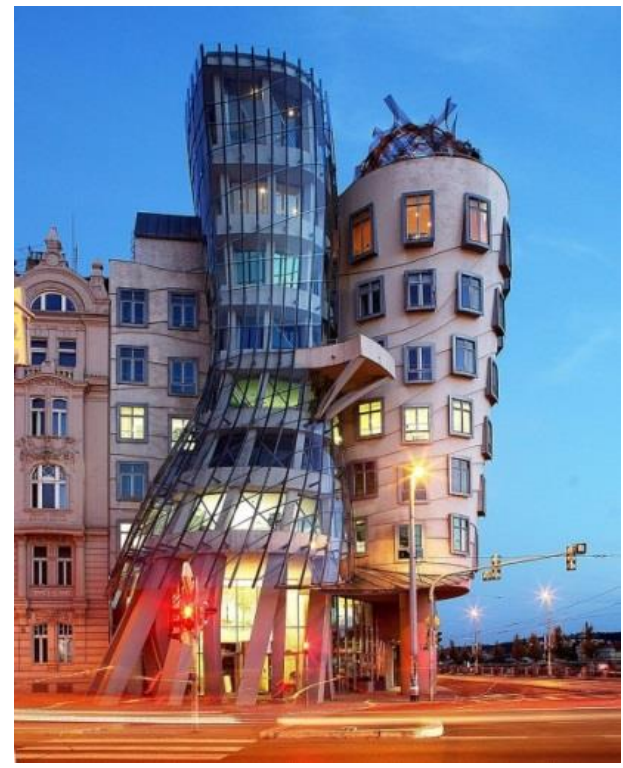

Fig. 31 Dancing House, Prague (Gehry, 1996)

It should also be noted that figures in these buildings are derived from fractal geometry, as well as from non-Euclidean geometries, as in the architectural realizations of Gehry and Libeskind (Figures 33 and 34).

Notice that this part of Mathematics goes beyond the traditional one (composed of Algebra and Geometry) and moves towards abstract contents, as presented by Topology. In this context, Mathematical Analysis enlarges its field of interest, just as Art and Architecture assume nonconventional aspects. 


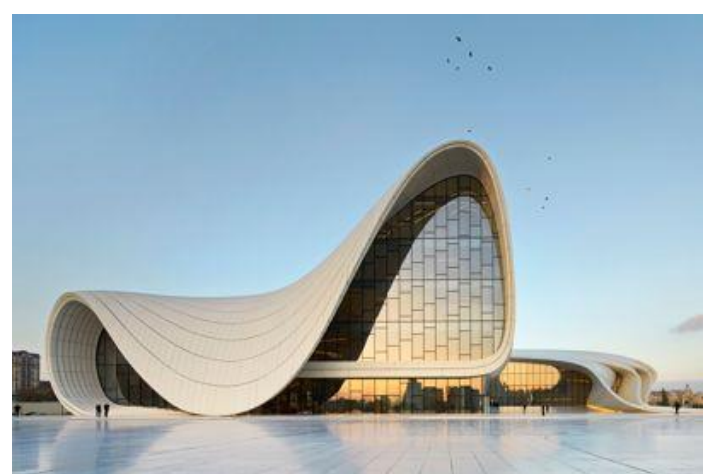

Fig. 32 Cristal Hall, Baku (Hadid, 2012)

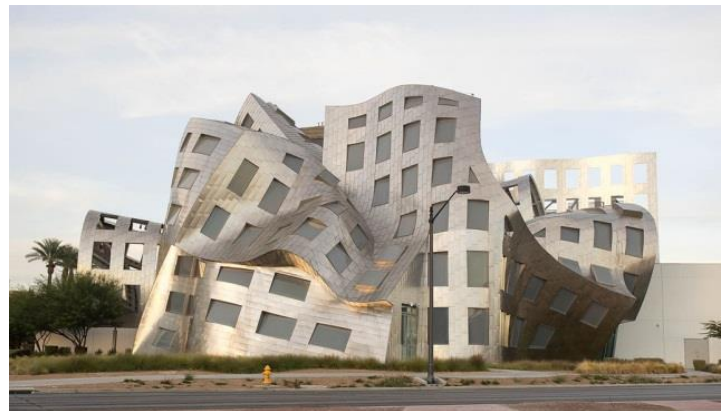

Fig. 33 Centre for brain health, Las Vegas ( Gehry, 2010)

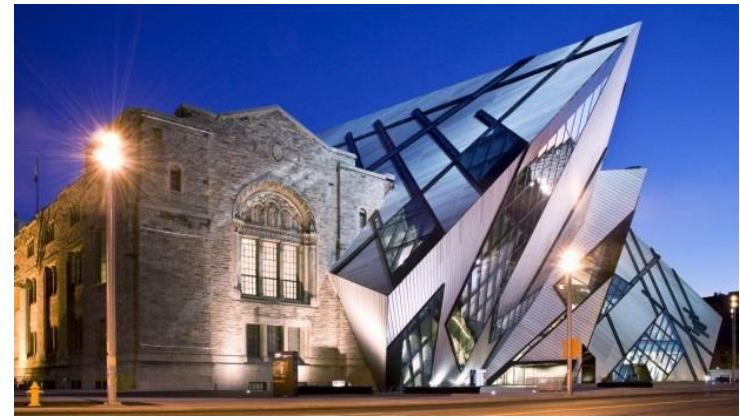

Fig. 34 Royal Ontario museum, Toronto (Libeskind, 2007)

\section{CONCLUSION}

The aim of this paper is to emphasize the link between sciences and humanities, recognizing that a unified culture persisted until the end of the $18^{\text {th }}$ century and the beginning of the $19^{\text {th }}$. An important demonstration of this is given by architects and physicians, who studied Mathematics and Physics at the time, at the école preparatoire, before entering their proper universities. Unfortunately, after this period the situation changed, particularly in Italy, where those studying Mathematics and Philosophy, like Peano, Enriques, etc., became marginal in the diffusion of culture.

Another aspect deals with the connections among Architecture, Mathematics and Philosophy, as studied mainly in continental Europe. Such connections can represent attempts towards a theory which interprets modern architectural realisations through mathematical and philosophical ideas.

Obviously, these are only attempts, but culture develops one step at a time, overcoming its errors, as stated by Engels in Antiduring (Engels, 1971). A new proposal could be a philosophy of Geodesy and Geomatics. Indeed, since geodetic and geomatics contents are becoming more and more immaterial, it is conceivable that Geodesy and Geomatics, besides having close relations with Mathematics and Physics, can meet Human Sciences.

\section{REFERENCES}

Badiou, A., 2017. Elogio delle matematiche. Mimesis, Milano-Udine.

Borzacchini, L., 2015. Storia e fondamenti della matematica. www.dm.uniba.it/Members

Capra, F. and Mattei, U., 2017. Ecologia del diritto. Aboca Edizioni, Sansepolcro (Arezzo).

Cassirer, E., 2015. I problemi filosofici della teoria della relatività. Mimesis, Milano-Udine.

Debuiche, V., 2012 Leibniz's Manuscripts on Perspective. 5th international conference of the European Society for the History of Science, Nov 2012, Athens, Greece.

Dellapiana, E. and Montanari, G. (2017). Una storia dell'architettura contemporanea. Utet, Novara.

Gombrich, E.H., 1950. La storia dell'arte. Einaudi, Torino

Engels, F., 197. Antiduring. Editori Riuniti, Roma.

Holdane, J., 1999. Form, meaning and value: a history of the philosophy of architecture. The Journal of Architecture. Vol. 4 (1), pp 9-20.

Koyré, A., 1967. Dal mondo del pressappoco all'universo della precisione. Einaudi, Torino.

Odifreddi, P., 2011. Una via di fuga. Mondadori, Milano.

Panofsky, E., 1961. La prospettiva come forma simbolica ed altri scritti. Feltrinelli, Milano.

Panofsky, E., 2016. Gothic Architecture and Scholasticims. Abscondita, Milano.

Rovelli, C., 2014. Sette brevi lezioni di fisica. Adelphi, Milano.

Rovelli, C., 2017. L'ordine del tempo. Adelphi, Milano.

Sala, N. and Cappellato, G., 2003. Viaggio matematico nell'arte e nell'architettura. Franco Angeli, Milano.

Stewart, I., 2016. Numeri incredibili. Bollati Boringhieri, Torino.

Zellini, P., 2016. La matematica degli dei e gli algoritmi degli uomini. Adelphi, Milano.

Zoja, L., 2003. Storia dell'arroganza, Moretti e Vitali, Bergamo. 